\title{
Effectiveness of non-pharmaceutical interventions on cognitive function among non-demented African American and Latino older adults in the USA: A scoping review
}

\author{
Ashley Shaw \\ University of Kansas Alzheimer's Disease Center https://orcid.org/0000-0003-2018-7274 \\ Jaime Perales-Puchalt ( $\nabla$ jperales@kumc.edu ) \\ https://orcid.org/0000-0003-1013-7847 \\ Esmeralda Valdivieso-Mora \\ University of Kansas \\ Jerrihlyn L. McGee \\ University of Kansas Medical Center \\ Prasanna Vaduvathiriyan \\ University of Kansas Medical Center \\ Eric D. Vidoni \\ University of Kansas Alzheimer's Disease Center
}

\section{Research article}

Keywords: dementia, minorities, aging, cognitive training, exercise, diet, older adult, clinical trial

Posted Date: September 24th, 2019

DOl: https://doi.org/10.21203/rs.2.14918/v1

License: (c) (i) This work is licensed under a Creative Commons Attribution 4.0 International License. Read Full License

Version of Record: A version of this preprint was published at Ethnicity \& Health on October 6th, 2020. See the published version at https://doi.org/10.1080/13557858.2020.1828292. 


\section{Abstract}

Objective African Americans and Latinos/Hispanics have a higher prevalence of dementia compared to non-Latino Whites. This scoping review aims to synthesize non-pharmaceutical interventions to delay or slow age-related cognitive decline among cognitively healthy African American and Latino older adults.Design A literature search was performed in PubMed, CINAHL, PsycINFO and Web of Science between January 2000 - May 2019. Relevant cited references and grey literature were also reviewed. Four independent reviewers evaluated 1,181 abstracts, and fullarticle screening was subsequently performed for 145 articles. The scoping review consisted of eight studies, which were evaluated according to peer-reviewed original manuscript, non-pharmaceutical intervention, cognitive function as an outcome, separate reporting of results for African American and Latino individuals, minimum age of 40 , and conducted in the United States. A total of 8 studies were considered eligible and were analyzed in the present scoping review.

Results Eight studies were identified. Four studies focused on African Americans and four focused on Latino individuals. Through the analysis, results indicated cognitive training-focused interventions were effective in improving memory, executive function, reasoning, visuospatial, psychological function, and speed among African Americans. Exercise interventions were effective in improving cognition among Latino individuals.

Conclusion This scoping review identified effective non-pharmaceutical interventions among African American and Latino individuals. Effective interventions focused on cognitive training alone for African Americans and exercise combined with group educational sessions for Latino individuals. Future research should explore developing culturally appropriate non-pharmaceutical interventions to reduce disparities and to enhance cognition among older African American and Latino individuals.

\section{Introduction}

Increased life expectancy in the United States has fueled an unprecedented growth in the prevalence of dementia. Approximately 5.4 million Americans have dementia and the prevalence is expected to double by 2050.[1] Dementia is one of the major causes of mortality and disability in later life and has a significant health impact on family members and caregivers.[2-4] The most common type of dementia, Alzheimer's Disease, is now the most expensive disease in the U.S., more costly than cancer and heart disease, with the majority of those costs ( \$259 billion) paid by Medicare and Medicaid.[1] In addition, Mild Cognitive Impairment, a risk factor for dementia, has a yearly conversion rate to dementia of $5-10 \% .[5,6]$ In the absence of effective disease-modifying medications there is now a major effort to prevent cognitive decline or delay the onset of AD in our growing population of older adults, in particular through behavioral interventions such as diet and exercise.

A recent systematic review stated that encouraging, although inconclusive, evidence supports that lifestyle interventions may delay or slow age-related cognitive decline.[7] This report found the strongest evidence came from cognitive training and physical activity interventions based on randomized controlled trials (RCTs). Two years earlier, a less strict systematic review of 17 non-pharmaceutical RCTs found that from a variety of intervention modalities that included nutrition and supplementation, cognitive training and physical activity, the latter having specific favorable results on cognition.[8]

The high prevalence of dementia and cognitive impairment among ethnic minorities in the United States contrasts with their underrepresentation in clinical trials. For example, African Americans and Latino/Latina/Latinx/Hispanic individuals (Latino) have a prevalence rate that is 1.5 times higher than that of non-Latino Whites.[1] However, 
systematic reviews indicate that their inclusion in trials is minimal, which makes extrapolating results to these groups impossible.[7, 9-11] To date, no studies have attempted to identify interventions to reduce dementia risk among African American and Latino individuals. For this reason, we sought to synthesize the available evidence of the nonpharmaceutical interventions for age-related cognitive decline among cognitively healthy African American and Latino older adults. Identifying these interventions is important, as effective interventions for a majority non-Latino White population do not necessarily translate into effective interventions for other racial/ethnic groups. Results from this review inform the development of culturally-sensitive risk-reduction interventions for dementia.

\section{Methods}

\section{Search Strategy}

We performed a literature search between May 1-May 7 in 2019 in PubMed, CINAHL, PsycINFO and Web of Science. We also searched for relevant cited references and grey literature. A combination of keywords and controlled vocabulary terms were used to identify the non-pharmaceutical interventions in older African American and Latino populations. The search was limited to studies from January 1, 2000 to April 30, 2019 by applying the databases' publication date limit feature. No language restrictions were applied to the search. The search strategy resulted 1,178 unique results after de-duplicating using the bibliographic software EndNote (Endnote X8, Clarivate Analytics, Clarivate.com). A detailed search strategy is available in Appendix 1.

\section{Study Selection}

We screened for original studies that reported on non-pharmaceutical interventions with a cognitive function outcome among non-demented African American and Latino populations. The criteria for inclusion were: 1) peer reviewed original manuscripts including 2) non-pharmaceutical intervention trials, independent of the number of intervention arms, 3) with cognitive function as an outcome, 4) reporting results for African Americans and/or Latino individuals separately, 5) with a minimum age of 40 , and 6) conducted in the United States. The literature review was conducted in three phases. Phase 1 consisted of a double screening of all titles and abstracts by four authors (AS, JP, EVM, and EDV). Reviewers used a duplicate-free library to independently review each title and abstract and excluded non-related ones. For Phase 2, the same four authors double-reviewed the full texts of the positively screened articles to further exclude those not fulfilling the eligibility criteria. Phase 3 consisted of the data extraction, conducted by two of the authors (ARS, and EDV).

\section{Data Extraction and Synthesis}

Data were extracted into a spreadsheet with the following information: first author, year, city and state of intervention, setting of intervention, demographic and clinical characteristics of participants, acculturation assessment, type of trial, specific ethnoracial minority group(s), components of the intervention, intervention nature, cultural and individual tailoring, modality of the intervention, operationalization of cognitive function, and main findings. In case of discrepancy, if no agreement was met by readers after discussion, a majority decision was made. This review followed a standard framework for conducting scoping reviews.[12]

\section{Results}


A total of 2,164 articles were identified through electronic databases (PubMed, CINAHL, PsychINFO, and Web of Science), relevant cited references, and grey literature. Three manuscripts were added manually. In total, 1,181 articles were identified after duplicates were removed. After review of abstracts, an additional 1,036 articles were excluded. Of the 145 articles reviewed; 137 were excluded for one or more of the these reasons: 1 ) the study was not a peer reviewed original manuscript $(n=22), 2)$ the study was not a trial $(n=30), 3)$ the treatment was not nonpharmaceutical $(n=20), 4)$ the study was not conducted in the United States $(n=14), 5)$ did not report the results for African Americans and/or Latinos individuals separately $(n=62), 6)$ included individuals < 40 years old $(n=42), 7)$ included individuals who were not cognitively normal at baseline $(n=94)$, and 8) cognition was not a measured outcome $(n=95)$. A total of 8 articles were eligible for inclusion. Study selection flow diagram is shown in Figure 1.

Two types of trial designs were identified across the 8 studies, including randomized control trials $(n=7)$ and single group pre-post trials $(n=1)$. Among the randomized controlled trials, all studies randomized to two groups except the ACTIVE trial that included three groups.[13] An additional file shows a brief summary of the study characteristics and main outcomes presented in Table 1 [see Additional file 1: Table 1].

\section{Overview of Characteristics of Participants in Included Studies}

A total of 3,928 participants were recruited across the 8 studies examined in the scoping review. Five studies reported specifically on Latino individuals ( $n=1,258)$, and three reported specifically on African Americans $(n=1,015)$. Participants' age ranged from 55 to 94 years. Participants were predominately women $(76.8 \%-100 \%)$ across all eight studies. Among studies that reported geographical area $(n=5)$, all reported taking place in urban areas in Illinois $(n=$ $2)$, California $(n=2)$, and New York $(n=1)$. Studies that reported the setting $(n=6)$ varied from community centers ( $=2)$, senior centers $(n=3)$, to public schools $(n=1)$. One study that took place at a senior center also reported use of additional settings including a university wellness clinic and an apartment complex for low-income older adults. Lastly, four studies reported acculturation with regards to preference of language in the delivery of the interventions. Among these studies $(n=3) 33.3 \%, 73.0 \%$, and $96.4 \%$ of participants reported Spanish as their preference.

\section{Focus and Outcomes of Included Studies}

All studies assessed the cognitive domain of memory as an outcome. Also, several studies assessed additional cognitive domains including reasoning, speed, executive function, and global cognition. Instruments used to assess cognition varied for each study. The most common instruments used to assess cognitive function were Modified Mini-Mental State $(n=2)$, Trail Making Test $(n=2)$, and the Rivermead Behavioral Memory Test $(n=2)$.

Over half of the interventions (57\%) included some form of exercise. Of the exercise-focused interventions $(n=4)$, the target population in all was Latino individuals and the study designs included randomized control trials $(n=3)$ and a single group design $(n=1)$. Specifically, two studies reported using dance sessions, which showed improvement in executive function [14] and global cognition [14, 15] among participants. Also, two studies reported using structured exercise training in the interventions,[ in which one resulted in higher cognitive function scores [16] and one showed promise of having an positive impact on cognitive function in older Latino individuals.[17] Of the studies that examined executive function, participants in the intervention groups performed significantly better compared to the control group.[14, 18].

Interventions in seven studies were delivered using in-person group sessions and one study indicated using both group and individual cognitive exercises as part of the intervention.[13] Duration and intensity of interventions varied 
across examined studies. Duration of interventions ranged from four weeks to 36 months. Intensity of study interventions varied from 1-hour sessions $(n=5), 1.5$-hour sessions $(n=1)$, to 2.28 hour sessions $(n=1)$. Lastly, most of the studies examined were tailored to culture $(n=4)$. Of the tailored studies, two were developed considering Latino's appreciation of dancing and one was tailored first through focus groups to incorporate important cultural themes, [ Six (75\%) of the studies examined reported language of intervention, in which most were delivered in both English and Spanish $(n=3)$, followed by English only $(n=2)$, and Spanish only $(n=1)$.

\section{Characteristics of Included Studies focused on African Americans}

Four studies primarily included African Americans within their sample. However, one of the four studies had a relatively small sample of African Americans $(11 \%, n=29)$.[19] Of the three studies that had larger sample sizes,[ participants were more likely to be female with low education and income. Acculturation was not assessed in any of the four studies that focused on African Americans.

Recruitment efforts varied across studies focused on African Americans. However, most studies indicated recruiting African Americans via churches and senior centers or senior targeted events.[18-20] Use of local media such as TV and print was also a recruitment tool found to be successful in reaching older African Americans.[18] All studies examined required African American participants to have no communication or vision problems. Further, most studies examined in this review indicated that individuals were excluded for having a history of conditions such as cancer or stroke.

Three of the four studies that specifically examined African Americans involved cognitive training as an intervention, but varied in terms of type of cognition measured ranging from memory (verbal and nonverbal), executive function, reasoning, visuospatial, psychological function and speed.[13,18, 19] Also, execution of the cognitive training varied across studies including group training $[18,19]$ and a combination of individual and small group training.[13] The fourth study focused on the consumption of vitamin D and its effects on cognition among African Americans in which types of cognition measured included memory, language, and orientation. [20]

Of the interventions focused on African Americans, 3 of the 4 studies reported increasing cognitive performance from baseline to follow-up. When compared to other racial/ethnic groups, the cognitive performance of African Americans was found to be higher in the memory domain compared to Latino individuals, and non-Latino Whites.[19] One study, however indicated that the intervention was only slightly successful for supporting cognition in African Americans due to racial disparities found within memory and reasoning training gains compared to non-Latino Whites.[13] It is important to note that even though this study did not show an effect of the intervention on cognition among African Americans, there was an improvement in reasoning and memory performance in this group.

The main limitations of the examined non-pharmaceutical intervention studies that focused on African Americans were their small sample size, generalizability, and short intervention duration. Examined studies reported a relatively small sample size of African Americans within the intervention group.[13,18] There was a lack of socioeconomically and educationally diverse African Americans represented reported in the examined studies and participants were more likely to be female, resulting in a low generalizability among African Americans.[13, 18-20] Due to the short duration of most of the non-pharmaceutical interventions focused on African Americans, researchers were unable to determine if the intervention program could both enhance and maintain cognition over time among African Americans.[13] 


\section{Characteristics of Included Studies Focused on Latino individuals}

Of the eight included studies, four focused on interventions for Latino individuals. Acculturation was operationalized through focus groups that identified aspects of culture important to the Latino community or by delivering the intervention in Spanish. Three of the four examined studies indicated that at least $73 \%$ of participants preferred Spanish over English for intervention delivery.[14, 16, 17] Recruitment efforts for Latino individuals were primarily through senior centers [15-17] and through established relationships from the lead investigator.[14, 15]

Studies examined required Latino participants to be currently engaged in limited physical activity. Most studies excluded individuals currently engaging in regular physical activity or having health conditions/diseases such as a stroke or recent bone fracture. All four of the examined studies indicated that interventions were group-oriented, held in person, and included a physical activity component. Cognitive outcomes varied in terms of domain measured, ranging from memory (verbal and nonverbal), executive function, reasoning, visuospatial, psychological function and speed. All four of the intervention studies among Latinos were found to be effective in improving cognitive performance.[16, 17]

The main limitations of the non-pharmaceutical Latino-focused intervention studies were sample size, generalizability, too few measures of cognition, control group treatment, and not assessing intensity of exercise activity in relation to cognition. Two examined studies reported small sample sizes, which may have impacted the ability to identify exercise related improvements in other cognitive domains.[14, 15] Due to recruitment strategies being targeted at public institutions such as senior centers, and interventions being carried out at a single site, results may not be generalizable to Latino individuals in other settings including primary care.[14, 17] Two studies reported assessing and analyzing too few measures of cognition, and reported including more measures may have been helpful to better understand the effect of the intervention.[16, 17] Of the studies examined that included a control group, two indicated that the control groups were not truly untreated and got some type of intervention such as health education.[14, 16] Lastly, interventions incorporating exercise did not examine intensity of exercise in relation to cognition, as intensity of exercise may have impacted cognition.[14]

\section{Similarities/Differences Between African American and Latino Studies}

Among the studies examined there were several similarities and differences found between African American and Latino individuals. Recruitment efforts were found to be similar between African American and Latino individuals, in

which participants were primarily recruited through senior centers. However, African Americans were more likely to be recruited through churches than Latinos.

Seven of the eight interventions focused on African American and Latino individuals were found to be effective in improving cognition from baseline to follow-up. The biggest difference between interventions aimed at Latino individuals compared to African Americans was the intervention modality. All interventions for Latino individuals involved some type of physical activity and were all group oriented. In contrast, most interventions aimed at African Americans focused on cognitive training. Only one study with African Americans focused on a dietary intervention to improve cognition. Also, interventions for Latino individuals incorporated cultural activities within the intervention design such as dancing, and took into account acculturation (language) within the delivery of the intervention, which was not seen among intervention studies for African Americans. 
Although all interventions measured cognition, non-pharmaceutical interventions for African Americans incorporated more measures of cognition across examined studies compared to interventions for Latinos. Similar reported limitations of examined studies included sample size and generalizability. Differences in reported limitations were also found. Most African American-focused studies reported short duration of the intervention as a main limitation while examined studies focused on Latinos reported few measures of cognition and having a treated control group as main limitations.

\section{Discussion}

This review reports results from nearly 20 years of interventions aimed at improving cognition among older individuals including African American and Latino individuals in the United States. Our scoping review highlighted that these ethnoracial groups are underrepresented in randomized controlled trials aimed at improving cognitive function. Only eight non-pharmaceutical intervention studies have specifically reported outcomes among these underrepresented populations who are at elevated risk for cognitive decline and dementia. Interventions included dietary supplementation, cognitive training, and exercise in conjunction with group educational sessions. All were effective to some extent. Our review also revealed that most of the examined interventions were feasible, with adherence rates of $76 \%$ among African Americans and $66 \%-85 \%$ among Latino individuals. Cognitive trainingfocused interventions were effective in improving memory, executive function, reasoning, visuospatial function, and processing speed among African Americans. Exercise interventions were effective in improving cognition among Latino individuals. It is important to note that cognitive training intervention studies examined in this review encountered challenges in terms of sample size and generalizability, which is congruent with previous aging related research.

Despite the disproportionate incidence of dementia-related cognitive decline in these populations, limited studies have focused on non-pharmaceutical interventions to reduce risk of cognitive decline among African Americans and Latino individuals. The Latino-focused exercise interventions were both culturally and linguistically tailored and demonstrated acceptability, feasibility, and efficacy in improving cognitive performance. This finding is not only consistent with previous literature focused on increasing physical activity to reduce chronic health conditions, [ but also shows that culturally tailored exercise interventions for Latino individuals can effectively increase physical activity and enhance health outcomes.[21]

For African Americans, non-pharmaceutical interventions were cognitive training-focused and did not explicity incorporate cultural tailoring. Culturally adapted lifestyle interventions among African American older adults have been shown to be effective in increasing exercise and have been shown to be feasible to implement within the African American community.[22] Additionally, faith-based lifestyle interventions targeted to reduce chronic disease risks among African Americans have consistently shown to be effective at behavioral change.[23] Therefore, future research should explore how culturally tailored physical activities interventions with cognitive training can impact cognition among older African American adults.

It is important to note that only one of the studies incorporated a nutrition component,[ which has consistently been shown to impact brain function in previous research.[24, 25] Further, research has noted that unhealthy eating habits disproportionately impact minorities, resulting in chronic diseases and conditions such as obesity, type II diabetes, and hypertension, which are all associated with an increased risk of cognitive impairment.[26, 27] More nutritionbased interventions for non-dominant racial/ethnic groups are highly warranted to help improve both health outcomes and cognition. 
A few studies noted within the eligibility criteria excluded individuals who had certain conditions and diseases such as a stroke.[13,14] Since stroke rates are highest among African Americans, and the rate of strokes among Latino has increased since 2013,[ it is important to establish culturally appropriate inclusion/exclusion criteria to better reach and enhance diverse enrollment for African Americans and Latinos in non-pharmaceutical cognitive interventions.

All studies were conducted in urban areas and in various settings such as community and senior centers, suggesting there is lack of non-pharmaceutical intervention research being conducted in rural areas. There are currently 4.1 million African Americans and 2.7 million Latino individuals who reside in rural areas in the United States.[28] At the current growth rate it is projected that Latino individuals will be the largest minority group in rural areas of the United States by 2025 . Given that the rural Latino population continues to grow, it is important to reach this underserved population and address cognitive function by developing novel non-pharmaceutical interventions that are appropriate in reaching rural communities, as these individuals might have unique needs.

Because African American and Latino individuals do not represent a homogeneous group, future studies should explore non-pharmaceutical interventions on cognitive function in diverse groups (i.e. African Caribbean vs. AfroAmerican, Mexican vs. Puerto Rican, and first-generation immigrants vs. second-generation immigrants). Examining diverse subgroups of populations of African and Latino descent will lead to a richer understanding of how culture and acculturation influences health behaviors in these groups. Further, exploring diverse groups can help shape the customization of culturally sensitive non-pharmaceutical interventions designed to improve cognitive function.

Improved cognition performance as a result of cognitive training in studies assessed in this scoping review align with other non-pharmaceutical interventions that have focused on cognitive training through PC-based training and PCbased training combined with exercise. Computer-based cognitive training has been shown to stimulate immediate and delayed memory, and language in older adults.[29] PC game-based cognitive training has resulted in improvements of attention among older adults.[30] Research has also suggested that PC games designed to improve processing speed results in improvements in short-term memory and attention among older adults. [31] Since PC based cognitive training has shown promise, future research should explore how culturally tailored PC based training impacts cognition among African American and Latinos.

Similar to the Zahonde et al. [13] study in this scoping review which found that combining cognitive training with exercise improved memory, reasoning, and speed among African Americans; PC-based training combined with exercise has been shown to improve cognition among older adults of other ethnoracial backgrounds. Researchers conducted a randomized virtual reality-enhanced cycling exercise intervention among older adults and found that the cybercycling group achieved better cognition compared to individuals in the traditional cycling group.[32] Future research should explore exercise in combination with PC-based cognitive training intervention studies aimed at minorities to better understand its impact on change in cognitive domains.

This scoping review had several strengths including use of a comprehensive, systematic methodology searching in electronic databases and grey literature, conducted by a librarian. Despite the findings from this review, there are limitations that need to be taken into consideration when interpreting findings. We have excluded studies measuring dementia incidence rather than cognition. However, the chances of finding a study that assessed dementia incidence as an outcome among minorities is low given the length of the interventions in our findings. To optimize the search we only included studies involving African American and Latino individuals in the US. Including other ethnoracial groups in the US and abroad would have added a significant number of manuscripts to review with too small a potential to find additional studies. Given the small number of manuscript retrieved, it was not feasible to conduct a 
meta-analysis of the findings. Even though we did not reject literature in languages other than English, we did not search for manuscripts in other languages. However, we expect the chances of finding a manuscript in Spanish or other potential languages for research conducted in the United States to be low.

\section{Conclusion}

African American and Latino adults and older adults are underrepresented in non-pharmaceutical interventions for cognitive health. The results of the eight non-pharmaceutical interventions reviewed in this study hold promise in promoting cognitive improvement among African Americans and Latino individuals. In this review, we have summarized the effectiveness of non-pharmaceutical intervention studies among African Americans and Latino individuals. Effective interventions focused on cognitive training and diet for African Americans and exercise combined with group educational sessions for Latino individuals. Culturally-driven interventions were effective in improving cognition. Given the increase in racial/ethnic diverse groups in the U.S. and their underrepresentation in clinical studies, there is a need to expand culturally appropriate non-pharmaceutical interventions to reduce health disparities and to enhance cognition among older African American and Latino individuals.

\section{Declarations}

\section{Ethical approval and consent to participate}

Not applicable

\section{Consent for publication}

Not applicable

\section{Availability of data and materials}

All data analyzed during this study are included in this published article and its supplementary information files.

\section{Conflict of Interest}

The authors declare that they have no competing interests.

\section{Funding}

The scoping review was funded through the P30AG035982 project lead by the University of Kansas Alzheimer's Disease Center.

\section{Description of Author Roles}

Jaime Perales Puchalt designed the study. Prasanna Vaduvathiriyan designed and conducted the literature searches. Ashley Shaw, Jaime Perales Puchalt, Esmeralda Valdivieso-Mora and Eric Vidoni carried out the reviews independently. Ashley Shaw and Esmeralda Valdivieso-Mora extracted and analyzed the data. Ashley Shaw and 
Jaime Perales Puchalt drafted the paper. Eric Vidoni and Jerrihlyn McGee supervised the study and the writing of the paper. All authors provided substantive edits, read and approved the final manuscript.

\section{Acknowledgement}

Not applicable.

\section{References}

1.Association As: 2017 Alzheimer's disease facts and figures. Alzheimer's \& Dementia 2017, 13(4):325-373.

2.Lopez AD, Mathers CD, Ezzati M, Jamison DT, Murray CJ: Global and regional burden of disease and risk factors, 2001: systematic analysis of population health data. The Lancet 2006, 367(9524):1747-1757.

3.Sallim AB, Sayampanathan AA, Cuttilan A, Ho RC-M: Prevalence of mental health disorders among caregivers of patients with Alzheimer disease. Journal of the American Medical Directors Association 2015, 16(12):1034-1041.

4.Organization WH: Dementia: a public health priority: World Health Organization; 2012.

5.Mitchell AJ, Shiri-Feshki M: Rate of progression of mild cognitive impairment to dementia-meta-analysis of 41 robust inception cohort studies. Acta Psychiatrica Scandinavica 2009, 119(4):252-265.

6.Petersen RC, Caracciolo B, Brayne C, Gauthier S, Jelic V, Fratiglioni L: Mild cognitive impairment: a concept in evolution. Journal of internal medicine 2014, 275(3):214-228.

7.National Academies of Sciences E, Medicine: Preventing Cognitive Decline and Dementia: A Way Forward: National Academies Press; 2017.

8.Barnett J, Bahar-Fuchs A, Cherbuin N, Herath P, Anstey K: Intervention to prevent cognitive decline and dementia in adults without cognitive impairment: A systematic review. J Prevent Alzheimers Dis 2015, 2:38-44.

9.Zhou Y, Elashoff D, Kremen S, Teng E, Karlawish J, Grill JD: African Americans are less likely to enroll in preclinical Alzheimer's disease clinical trials. Alzheimers Dement (N Y) 2017, 3(1):57-64.

10.Faison WE, Schultz SK, Aerssens J, Alvidrez J, Anand R, Farrer LA, Jarvik L, Manly J, McRae T, Murphy GM, Jr. et al: Potential ethnic modifiers in the assessment and treatment of Alzheimer's disease: challenges for the future. Int Psychogeriatr 2007, 19(3):539-558.

11.Watson JL, Ryan L, Silverberg N, Cahan V, Bernard MA: Obstacles and opportunities in Alzheimer's clinical trial recruitment. Health Aff (Millwood) 2014, 33(4):574-579.

12.Colquhoun HL, Levac D, O’Brien KK, Straus S, Tricco AC, Perrier L, Kastner M, Moher D: Scoping reviews: time for clarity in definition, methods, and reporting. J Clin Epidemiol 2014, 67(12):1291-1294.

13.Zahodne LB, Meyer OL, Choi E, Thomas ML, Willis SL, Marsiske M, Gross AL, Rebok GW, Parisi JM: External locus of control contributes to racial disparities in memory and reasoning training gains in ACTIVE. Psychol Aging 2015, 30(3):561-572. 
14.Marquez DX, Wilson R, Aguinaga S, Vasquez P, Fogg L, Yang Z, Wilbur J, Hughes S, Spanbauer C: Regular Latin Dancing and Health Education May Improve Cognition of Late Middle-Aged and Older Latinos. J Aging Phy Activity 2017, 25(3):482-489.

15.Marquez DX, Bustamante EE, Aguinaga S, Hernandez R: BAILAMOS: Development, Pilot Testing, and Future Directions of a Latin Dance Program for Older Latinos. Health Educat Behav 2015, 42(5):604-610.

16.Piedra LM, Andrade FCD, Hernandez R, Boughton SW, Trejo L, Sarkisian CA: The Influence of Exercise on Cognitive Function in Older Hispanic/Latino Adults: Results From the " inverted exclamation markCaminemos!" Study.

Gerontologist 2017, 57(6):1072-1083.

17.Hernandez R, Cheung E, Liao M, Boughton SW, Tito LG, Sarkisian C: The Association Between Depressive Symptoms and Cognitive Functioning in Older Hispanic/Latino Adults Enrolled in an Exercise Intervention: Results From the " inverted exclamation markCaminemos!" Study. J Aging Health 2018, 30(6):843-862.

18.Carlson MC, Saczynski JS, Rebok GW, Seeman T, Glass TA, McGill S, Tielsch J, Frick KD, Hill J, Fried LP: Exploring the effects of an "everyday" activity program on executive function and memory in older adults: Experience Corps. Gerontologist 2008, 48(6):793-801.

19.McDougall GJ, Jr., Becker H, Pituch K, Acee TW, Vaughan PW, Delville CL: Differential benefits of memory training for minority older adults in the SeniorWISE study. Gerontologist 2010, 50(5):632-645.

20.0wusu JE, Islam S, Katumuluwa SS, Stolberg AR, Usera GL, Anwarullah AA, Shieh A, Dhaliwal R, Ragolia L, Mikhail MB et al: Cognition and Vitamin D in Older African-American Women- Physical performance and Osteoporosis prevention with vitamin D in older African Americans Trial and Dementia. JAGS 2019, 67(1):81-86.

21.Bopp M, Fallon EA, Marquez DX: A Faith-Based Physical Activity Intervention for Latinos: Outcomes and Lessons. 2011, 25(3):168-171.

22.Gretebeck K, Green-Harris G, Ward E, Houston S, Skora T, Brown M, Means J, Gretebeck R: Cultural Adaption of a Lifestyle Exercise Interventio nfor Older African Americans: Research Results. Innov Aging 2018, 2(suppl_1):18-18.

23.Sattin RW, Williams LB, Dias J, Garvin JT, Marion L, Joshua TV, Kriska A, Kramer MK, Narayan KM: Community Trial of a Faith-Based Lifestyle Intervention to Prevent Diabetes Among African-Americans. J Community health 2016, 41(1):87-96.

24.Sofi F, Abbate R, Gensini GF, Casini A: Accruing evidence on benefits of adherence to the Mediterranean diet on health: an updated systematic review and meta-analysis. Am J Clin nutr 2010, 92(5):1189-1196.

25.Lourida I, Soni M, Thompson-Coon J, Purandare N, Lang IA, Ukoumunne OC, Llewellyn DJ: Mediterranean diet, cognitive function, and dementia: a systematic review. Epidemiology (Cambridge, Mass) 2013, 24(4):479-489.

26.Satia JA: Diet-related disparities: understanding the problem and accelerating solutions. J Am Dietetic Assoc 2009, 109(4):610-615.

27.Gottesman RF, Albert MS, Alonso A, Coker LH, Coresh J, Davis SM, Deal JA, McKhann GM, Mosley TH, Sharrett AR et al: Associations Between Midlife Vascular Risk Factors and 25-Year Incident Dementia in the Atherosclerosis Risk in Communities (ARIC) Cohort. JAMA Neurol 2017, 74(10):1246-1254. 
28.Population Change and Geography /https://www.ers.usda.gov/webdocs/publications/44570/29568_eib8full.pdf? $\mathrm{v}=41305]$

29.Miller KJ, Dye RV, Kim J, Jennings JL, O’Toole E, Wong J, Siddarth P: Effect of a computerized brain exercise program on cognitive performance in older adults. Am J Geriatr Psychiatr 2013, 21(7):655-663.

30.Whitlock LA, McLaughlin AC, Allaire JC: Individual differences in response to cognitive training: Using a multimodal, attentionally demanding game-based intervention for older adults. Comput Human Behav 2012, 28(4):10911096.

31.Szelag E, Skolimowska J: Cognitive function in elderly can be ameliorated by training in temporal information processing. Restor Neurol neurosci 2012, 30(5):419-434.

32.Anderson-Hanley C, Arciero PJ, Brickman AM, Nimon JP, Okuma N, Westen SC, Merz ME, Pence BD, Woods JA, Kramer AF et al: Exergaming and older adult cognition: a cluster randomized clinical trial. Am J Prevent Med 2012, 42(2):109-119.

\section{Tables}

Table 1. Characteristics of Non-Pharmacological Interventions on Cognitive Functioning Among Older African Americans and Latinos

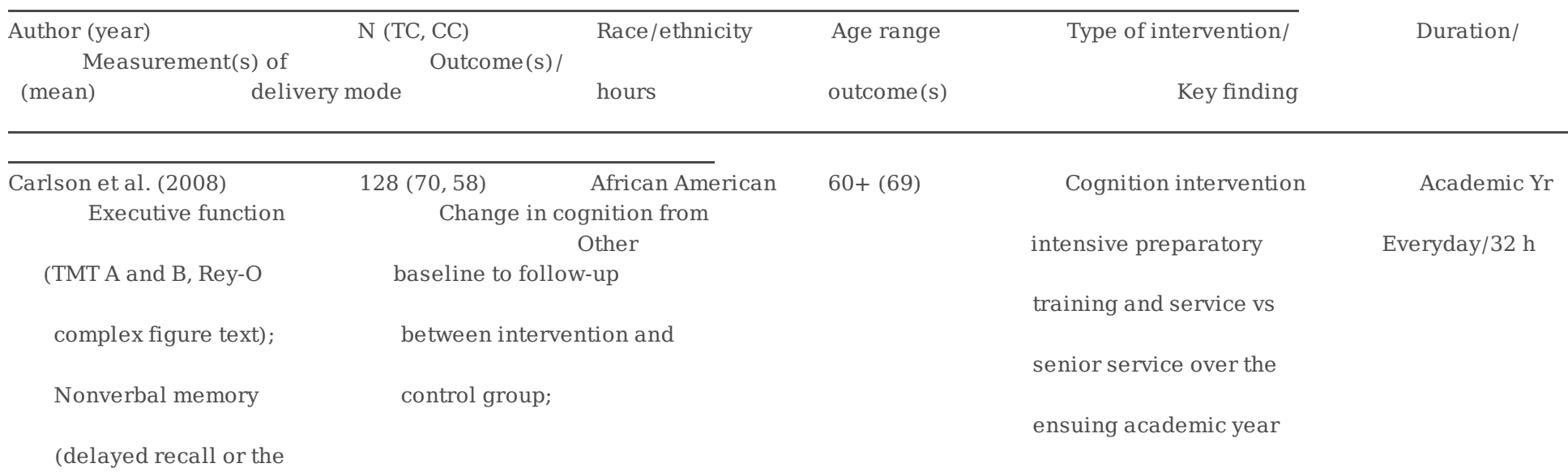

CFT following a 15-min

filled interval); verbal memory (three-learning trials with a list of 20 common words from the Iowa Established Populations for Epidemiologic Studies of the Elderly-Immediate and followed by a verbal recall)

Hernandez et al. (2018) 572

1h Modified Mini-Mental State

Cognitive measures

Hispanic/Latino NR (73.13)

(3MS) were

Scale (GDS)

associated with impaired

cognitive functioning among 
older adults in the exercise

intervention

Marquez et al. (2015)

$13(9, \mathrm{NR})$

Hispanic/Latino

$55-73(65.2)$

Dance sessions

13 weeks $/ 1 \mathrm{~h}$

East Boston Memory Test,

Change in test scores from pre to

In person, group sessions

perweek

Stroop

Neuropsychological post

post Screening Test, Numbers Comparison Test, Verbal Fluency

Marquez et al. (2017)

$57(28,29)$ $55-80$,
TC $(64.8)$,

Change in global and individual CC (66.4)

Making Test, Color task function;
Dance sessions

In person, group sessions $\quad 16$ weeks/ 1

per week

(Trail

from Stroop, Word fluency, Dance group performed symbol-digit modalities test); significantly better in executive Working memory (digit span function. Dance group and test, digit ordering test); CG improved in global cognition Episodic memory (logical memory I and II)

Table 1. Continued

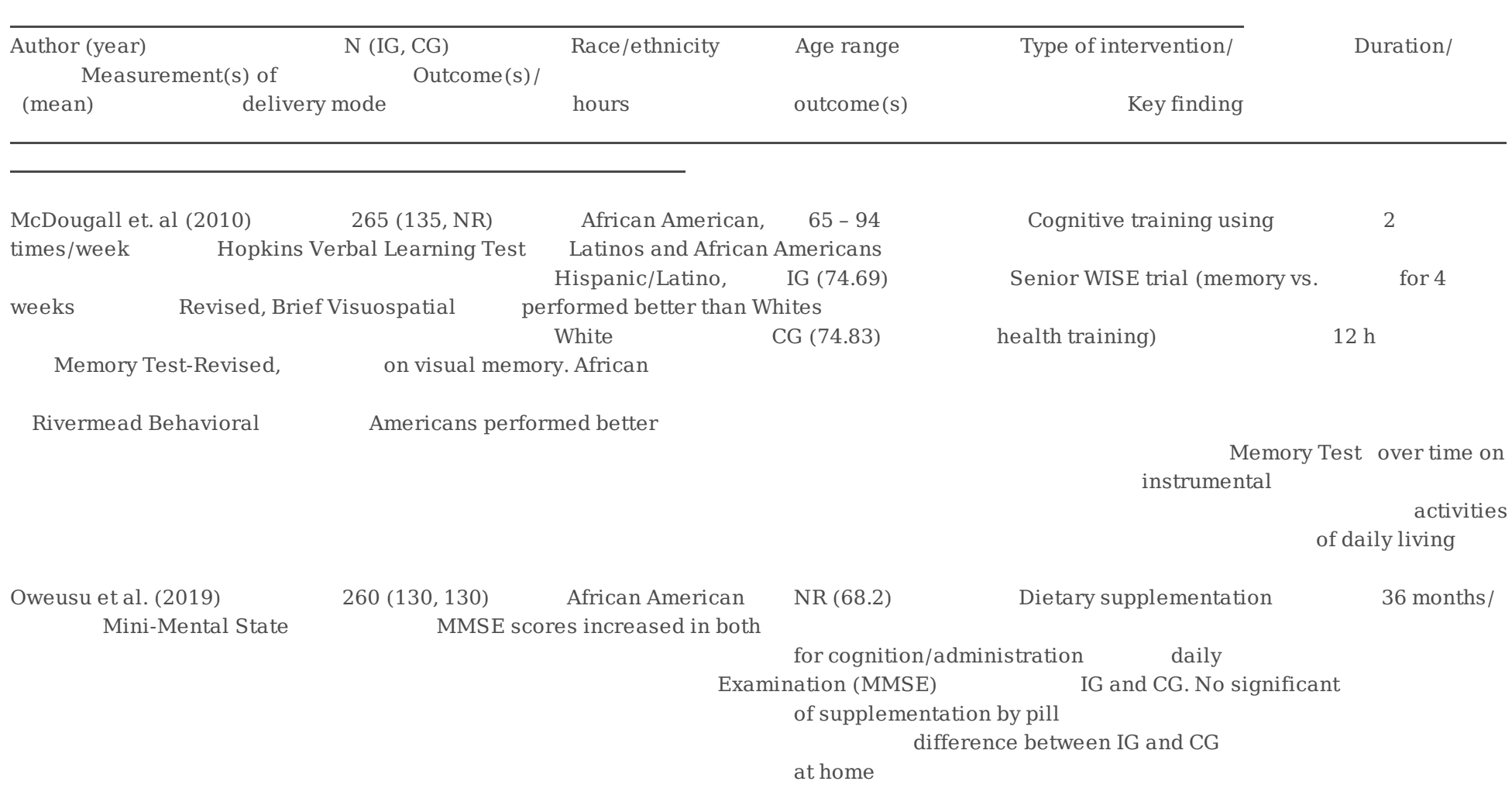




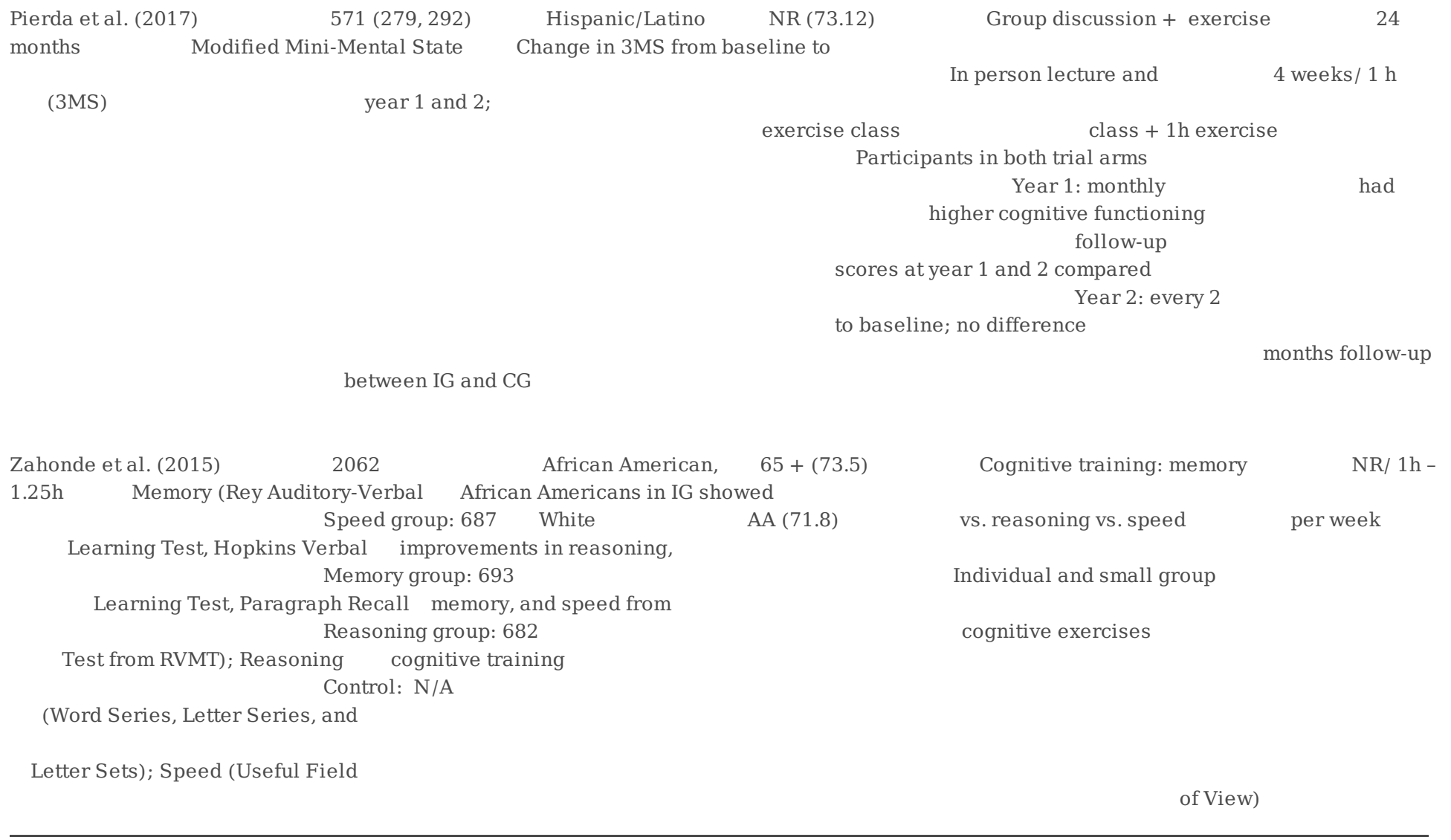

Note: $\mathrm{AA}=$ African American. $\mathrm{CG}=$ control group; IG = intervention group; NR = not reported

\section{Figures}


Fig. 1 Flow diagram

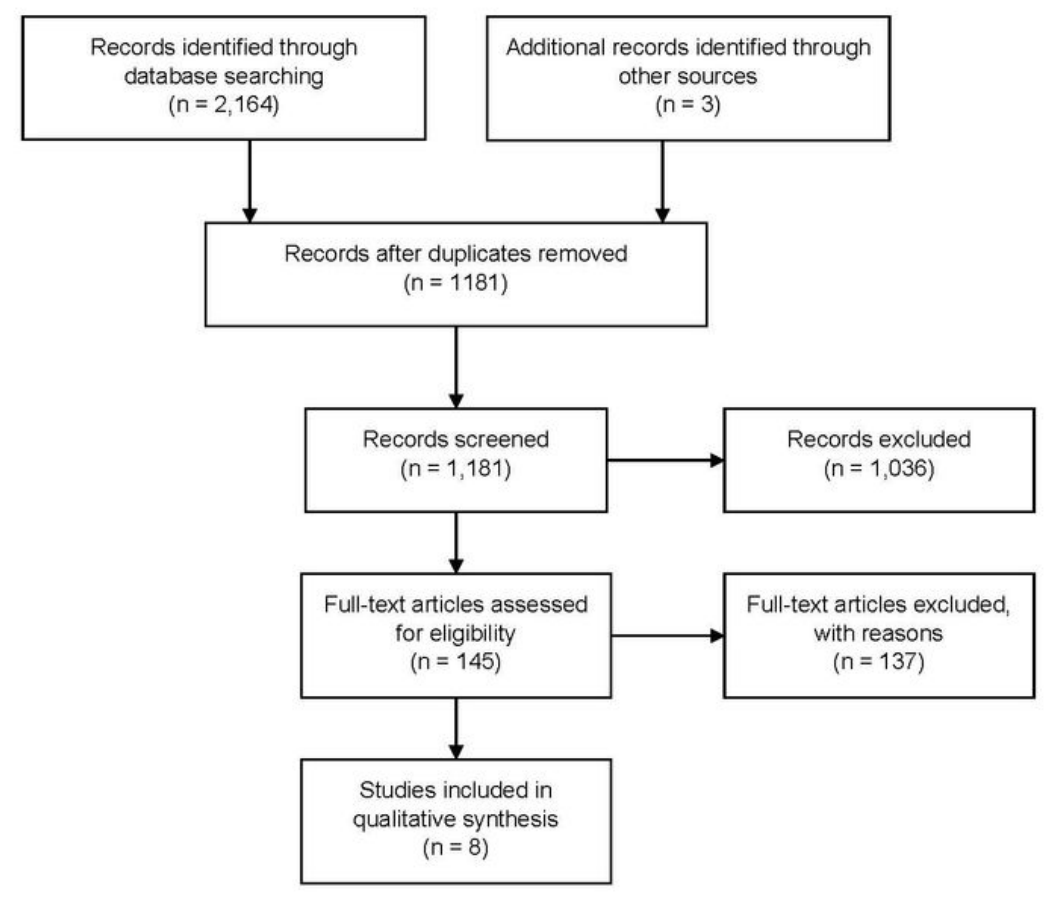

Figure 1

Flow diagram 